\title{
Las bibliotecas del sector salud
}

\section{Adolfo Rodríguez Gallardo, Director General de Bibliotecas e investigador del CUIB.}

\section{Antecedentes}

En 1982 el Maestro Álvaro Vázquez publicó el directorio de Bibliotecas en Ciencias de la Salud. El trabajo abarca 209 bibliotecas de este sector, tomándolo en su sentido más amplio: bibliotecas, centros de información, etc. Desde que se publicó el directorio, su aportación a la bibliotecología mexicana fue obvia, pues los datos que contiene revelan algunos aspectos importantes si se agrupan de diversas formas y se relacionan entre sí. El trabajo que presentamos a continuación está basado en el Directorio, no hubiera sido posible sin contar con tan importante obra.

\section{objetivo}

Pretendemos analizar la información disponible de manera que los directivos de bibliotecas y funcionarios públicos que tienen bajo su responsabilidad servicios bibliotecarios puedan tomar las medidas necesarias para la optimización de los servicios. Deseamos también conocer cómo está compuesto el sistema de bibliote- cas del sector salud y con qué recursos cuenta.

\section{Limitaciones}

La mayor limitación del trabajo es que está basado exclusivamente en la información del Directorio. No se hizo ningún intento de completar la información ni se confrontó ésta con otras posibles fuentes; por lo tanto, se omiten algunas bibliotecas de importancia no incluidas en el Directorio.

Dicha obra contiene bibliotecas que, además de los materiales relacionados con la salud, poseen otros de diferente índole. Ignoramos si los datos cuantitativos se refieren exclusivamente a obras pertinentes al sector salud - bien incluye el total de las obras de la colección sin haber efectuado una depuración previa. Como ejemplo de esta incertidumbre mencionaremos la inclusión de algunas bibliotecas centrales universitarias, las de facultades de química, el CICH, INFOTEC, los Laboratorios Nacionales de Fomento Industrial, la secretaría de Trabajo y Previsión Social, así como varias escuelas agrícolas. Al no contar con tal seguridad, es posible que los datos relativos al sector salud estén distorsionados. 
Hipótesis

Partimos del supuesto de que las colecciones universitarias constituyen parte considerable del subsistema. Los sistemas de clasificación son variados y no responden a las necesidades de las bibliotecas del subsistema; por lo tanto sería óptimo capacitar al personal de estas bibliotecas en la clasificación del Sistema de la Biblioteca Nacional de Medicina de los Estados Unidos.

En el Distrito federal existe una gran concentración de todos los recursos. El desarrollo en la República, algunas regiones gozan de recursos importantes en tanto que otras carecen de ellos.

Metodología

Para facilitar el trabajo de análisis y presentar los resultados con mayor claridad hemos agrupado las bibliotecas en tres grandes bloques :

1.Bibliotecas oficiales; comprenden las de la secretaría de Salubridad y Asistencia, el Instituto Mexicano del Seguridad y Servicios sociales para los Trabajadores del Estado.

2.Bibliotecas universitarias; incluye a todas las que forman parte de una universidad, ya sea ésta pública o privada. Las bibliotecas fueron agrupadas de acuerdo al esquema de regionalización propuesto por la Asociación Nacional de Universidades e Instituciones de Educación Superior.

3.Bibliotecas diversas; las no observadas en los grupos anteriores, sin importar si se trata de un organismo público o privado.
Los datos referentes tanto al número total de bibliotecas como de libros, volúmenes encuadernados, etcétera, no coinciden con los del Directorio porque hay tres bibliotecas duplicadas, pues fueron incluidas como bibliotecas oficiales así como universitarias porque forman parte de escuelas de enfermería o de salud pública.

Resultados

El número total de bibliotecas es de 209, pero la cifra que aquí se maneja es 212. La diferencia son las tres bibliotecas mencionadas, para mayor facilidad duplicamos su contabilidad ya que la modificación de los datos no es significativa. Nuestra primera hipótesis -referente a la importancia de las bibliotecas que dependen de universidades-confirma que más de la mitad del total de bibliotecas pertenecen a esas instituciones.

Las bibliotecas universitarias localizadas en los estados son 76, representan una parte importante del porcentaje total, pues solamente un tercio, es decir 37, están en el Distrito Federal.

Por el contrario, las bibliotecas del sector oficial, tanto las de la Secretaría de Salud y Asistencia Pública como las del ISSSTE, se localizan exclusivamente en la ciudad de México. El Instituto Mexicano del seguro tiene una mejor distribución, cuenta con 15 bibliotecas que representan aproximadamente el $30 \%$ del total localizadas en el Distrito Federal.

En relación a las bibliotecas de instituciones diversas, sólo 5 de un total de 29, es decir el 15\%, están en provincia, en tanto que 
el 85\% de las bibliotecas diversas está en la Ciudad de México.

Es, pues, obvia la desproporcionada concentración de bibliotecas en la capital, llegándose en algunos casos a situaciones verdaderamente graves como Nayarit, Colima, Hidalgo, Tlaxcala, Campeche y Quintana Roo que carecen totalmente de bibliotecas en el sector salud.

En lo que se refiere a bibliotecas que no corresponden ni a universidades ni al sector salud, solo hay 5 en todo el país y corresponden a cada uno de los siguientes estados: Baja California Sur, Jalisco, Chiapas, Veracruz y Yucatán. Sin embargo en el Distrito Federal hay 24 bibliotecas de este tipo, cifra que representa el altísimo porcentaje del 85\%.

Los datos anteriores muestran que la distribución de bibliotecas sigue el patrón general del centralismo del que adolece nuestro país en todos los ámbitos.

Relación entre los distintos tipos de bibliotecas

La mayor parte de las bibliotecas del sector salud son universitarias ya que constituyen el 55\% del total frente a un 35\% en el sector oficial y un $10 \%$ en instituciones diversas. Lo anterior explica en parte que la mayoría de los empleados sean de bibliotecas universitarias, así como los mayores recursos bibliográficos.

Personal

La información contenida en el Directorio no nos permite distinguir el personal profesional del que no lo es; tampoco sabemos su grado de capacitación para la prestación de servicios o la realización de procesos técnicos, Todo el personal está englobado en una sola cifra. Los recursos humanos de las bibliotecas del sector salud suman 1,055 personas; de ellas, 627 trabajan en bibliotecas universitarias, cifra que representa más del 60\% del total de los recursos humanos del sector. El personal correspondiente a las bibliotecas del sector oficial es de 258 empleados, aproximadamente un $25 \%$ y unos 170 en instituciones diversas lo que equivale a un 17\%.

Como en todos los demás aspectos de la vida nacional, la mayor parte de los recursos humanos están ubicados en el Distrito Federal ya que casi el 60\% del personal adscrito a las bibliotecas del sector salud, 596 personas aproximadamente, prestan sus servicios en esa ciudad. Al analizar encontramos una adecuada distribución del personal de bibliotecas universitarias y su localización en las ocho regiones en que las ha dividido ANUIES, pues el Distrito Federal cuenta tan solo con un 32\% de los recursos humanos.

Por último, las instituciones diversas tienen una marcada concentración del personal en el Distrito Federal, aproximadamente casi el 90\% de los empleados de estas bibliotecas se encuentran en la capital; es decir, de un total de 170 personas, 148 laboran en instituciones localizadas en el Distrito Federal y sólo 22 en otros lugares.

\section{Libros}

El sistema cuenta con 755, 630 libros, cifra que abarca todos los tipos de bibliotecas. Sin embargo, 
la información del Directorio no nos permite saber cuántos de esos libros son títulos duplicados, cuántos materiales obsoletos, cuántos no pertenecen a obras relativas al sector salud, pues como ya hemos dicho, en él se incluyen algunas bibliotecas relacionadas con este sector sólo marginalmente. También sería importante saber qué proporción de estos materiales es de apoyo directo en actividades de investigación y cuál en actividades docentes.

La desproporción de los recursos bibliográficos, al menos en cantidad, es enorme. Las bibliotecas universitarias poseen 546,167 libros, cifra que representa un imponente $72.5 \%$, en tanto que el sector oficial solo dispone de 108,592 libros, equivalentes a un $14 \%$ del total de los recursos. Por último, las instituciones diversas cuentan con similares recursos bibliográficos a los del sector oficial, pues disponen de 100,871 libros en forma de monografía, es decir, el 13.5\% del total.

Al analizar el número de los libros que forman parte de las colecciones universitarias encontramos que la distribución, aunque desigual, guarda una proporción adecuada. Las siguientes columnas representan las cifras de los libros y regiones a las que pertenecen; los datos fueron tomados del cuadro de recursos.

$\begin{array}{ll}\text { REGIÓN } & \text { LIBROS } \\ \text { I } & 13,614 \\ \text { II } & 70,711 \\ \text { III } & 19,541 \\ \text { IV } & 34,748 \\ \text { V } & 24,843 \\ \text { VI } & 67,283 \\ \text { VII } & 12,240 \\ \text { VIII } & 303,187 \\ \text { TOTAL } & 546,167\end{array}$

Las colecciones, tanto de libros como de revistas, muestran una mayor concentración en la ciudad de México, aunque en menor grado, como número de bibliotecas y de personal empleado y otros factores.

Volúmenes encuadernados

Esto rubro fue incluido y contabilizado en el directorio, pero la información al respecto es ambigua. ¿Se trata de publicaciones periódicas encuadernadas cuando se completa un volumen? ¿Se refiere a volúmenes no completos pero encuadernados? Podría tratarse de libros y otro tipo de materiales como folletos? La duda hace que nos abstengamos de una interpretación sobre el tema.

Títulos de revistas

De todos los recursos con que cuentan las bibliotecas del sector salud, los títulos de revistas es el que guarda una proporción más armónica. Los recursos hemerográficos de las universidades, aunque siguen siendo mayores que en los otros sectores, no son tan desproporcionados si se toma en cuenta el gran número de usuarios a los que sirven. Las bibliotecas de las universidades cuentan con 14,555 títulos que significan un 49\% del total de los recursos; las bibliotecas del sector oficial suman 10,545 títulos equivalente a 35\% de las publicaciones periódicas; y por último, las instituciones diversas reciben 4,788 títulos que representan un 16\% de las revistas.

Al igual que con los libros, en este caso tampoco contamos con información pertinente que nos deje evaluar la calidad de los materiales recibidos. Sería interesante 
saber, por ejemplo, cuántos títulos se utilizan para apoyo a la enseñanza; cuántos para la investigación; cuántos están duplicados; cuántos no corresponden al área de la salud y están incluidos porque las instituciones a las que pertenecen fueron incluidas aunque no se dedique primordialmente a los aspectos relacionados con el sector salud.

Cabe destacar que una posible explicación a la alta cifra de títulos de revistas que poseen las bibliotecas del sector oficial, es que muchas de las bibliotecas corresponden a instituciones dedicadas a la investigación por lo que dependen en gran medida de publicaciones periódicas especializadas.

Sistemas de clasificación

Los sistemas de clasificación que se utilizan para organizar el material bibliográfico son muy variados. La mayoría de las bibliotecas usa el Sistema Decimal de Dewey y probablemente porque es el único traducido al español. El inconveniente es que la edición utilizada sea la decimoquinta, publicada en 1956, lo que nos hace pensar que la clasificación es obsoleta para ordenar los materiales bibliográficos de una disciplina sumamente cambiante. El 40\% de las bibliotecas utilizan este sistema pero, en el caso de las bibliotecas universitarias, la proporción aumenta pues 59 de las 113, es decir un 52\% del total, clasifican con el sistema Dewey.

La segunda clasificación en cuanto a su uso en bibliotecas es la elaborada por la National Library of Medicine. La usan 51 bibliotecas en total y sólo 4 de las 113 bibliotecas universitarias, aunque las del sector oficial sean las grandes consumidoras de este sistema con 44 bibliotecas que lo aplican. Casi el $80 \%$ de las bibliotecas que clasifican con el sistema de la National Library of Medicine corresponde al sector oficial. Es interesante señalar que las bibliotecas universitarias, quienes cuentan con mayores recursos en todos los aspectos, utilizan una clasificación poco adecuada a su tipo de acervo. También es importante destacar que hay 30 bibliotecas que usan clasificaciones propias o poco utilizadas por las demás o bien carecen de clasificación alguna; el porcentaje de estas bibliotecas es $30 \%$.

Valdría la pena hacer un esfuerzo para capacitar al personal dedicado a la clasificación en el uso del sistema de Biblioteca Nacional de Medicina de los Estados Unidos (National Library of Medicine), ya que cuenta con clasificación bastante actualizada y es el más adecuado para bibliotecas de biomedicina.

Bibliotecas universitarias

Las bibliotecas universitarias son las mejor distribuidas en el país. Tienen, salvo muy contadas excepciones, una auténtica cobertura nacional. Cuentan con los mayores recursos tanto en personal como en libros y títulos de revistas.

Todo proyecto que tienda a mejorar las bibliotecas del sector salud deberá tomar en cuenta a las bibliotecas universitarias. Es posible que su mayor deficiencia estribe en el uso de un sistema de clasificación de los materiales bibliográficos poco adecuado. Los recursos que las universidades poseen, si bien son los más impor- 
tantes, no bastan en relación al número de usuarios potenciales. Posiblemente la investigación que Martha Añorve está realizando sobre las bibliotecas universitarias del país nos permita conocer con mayor detalle los recursos y posibilidades de estas bibliotecas. Pero a la luz de la experien- cia de los recursos de estas bibliotecas cabe preguntarse, si las bibliotecas universitarias apoyan con sus recursos a otros sistemas bibliotecarios nacionales, como lo hacen entonces con el sistema bibliotecario del sector salud?

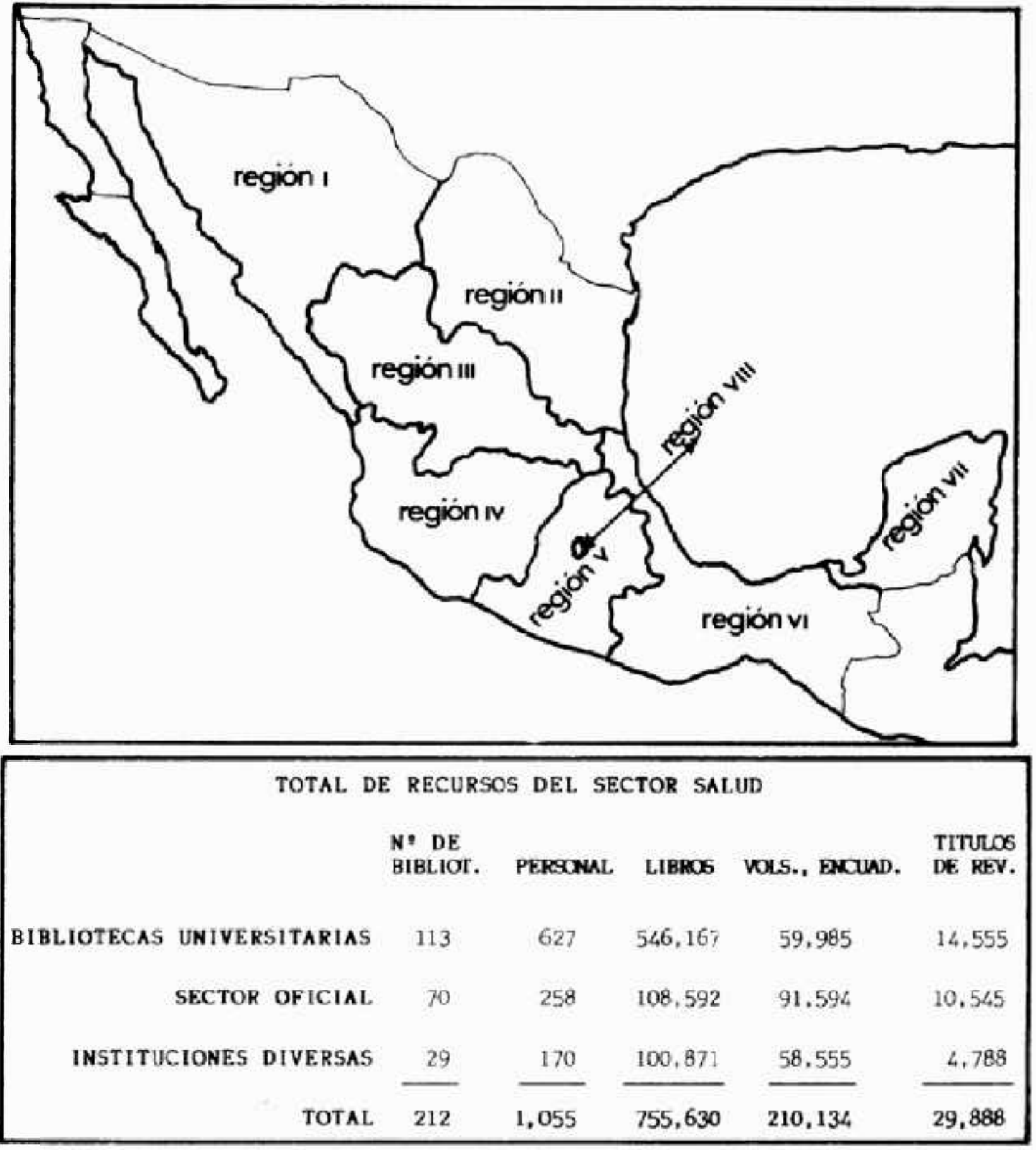




\section{TOTAL DE RECURSOS DE LAS OCHO REGIONES DE ANUIES BIBLIOTECAS UNIVERSITARIAS}

REGIONES DE ANUIES

REGION I

REGION II

REGION 1 :1

REGION IV

REGION $V$

REGION VI

REGION VII

REGION VIII

TOTAL
BIBLIOTECAS

PERSONAL

39

127

24

68

35

55

13

37

113
VOLUMENES ENCUADERNADOS
TITULOS Dâ REVISTA
714

244

28,311

6,551

3,216

8,318

2,087

640

79,872

129,709
1. 409

903

1,482

470

1,264

384

8,399
14,555

$266 \quad 303,187$

$627 \quad 546,167$

SISTEMAS DE CLASIFICACIONES USADOS

UNIVERITARIAS SECTOR

D D C

L C

N L M

OTROS

SIN CLASIFICACION

TOTAI
59

35

4

6

9

113
12

8

44

4

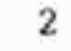

70
DIVERSAS

TOTAL

86

45

51

18

12

212

* Tres Bibliotecas se duplican, pues aunque están en el sector oficial tamicín son de escuelas. cono consecuencia si las restamos tendremos un total de 209 Bibliotecas. 


\begin{tabular}{|c|c|c|c|c|c|}
\hline BIRI.IOTECAS QUE SE ENCUENTRAN & $\begin{array}{l}\text { EN IS TH:1 } \\
\text { PERSUNAT. }\end{array}$ & FESIDADE: & $\begin{array}{l}\text { REGION I } \\
\text { VOLS., ENCUAD. }\end{array}$ & $\begin{array}{l}\text { TITULOS } \\
\text { DE REV. }\end{array}$ & CLASIF. \\
\hline \multicolumn{6}{|l|}{ CHIHUAIIUA } \\
\hline \multicolumn{6}{|l|}{ 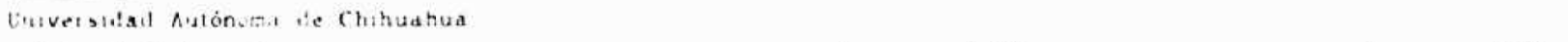 } \\
\hline kas. de Eutentweris & $?$ & 1.390 & & 4 & CDD \\
\hline Fac. de Ctancias Cuimicas & 3 & 2.491 & 200 & 20 & $C D D$ \\
\hline Fuc. de Metsecina & 2 & 2.133 & & 85 & (DI) \\
\hline TOTAL. DFL ESTADO & 7 & 6.014 & 200 & 109 & \\
\hline \multicolumn{6}{|l|}{ SWNORA } \\
\hline \multicolumn{6}{|l|}{ litiversidad de Soncro } \\
\hline tac. de Enfermeria y Trabajo Social & 2 & 978 & 6 & 30 & CDO \\
\hline TOTAL. DEL ESTADO & 2 & 978 & 6 & 30 & \\
\hline \multicolumn{6}{|l|}{ SINAIOA } \\
\hline \multicolumn{6}{|l|}{ Imversidad Autónoma de Sinaloa } \\
\hline Esc. de Ciencias del Mar & 2 & 839 & 225 & 6 & $C D D$ \\
\hline Fac. ie Cirncik Quimico-Piwidgicas & 3 & $1,5 \times 0$ & 10 & & \\
\hline Esc. He Trabajo Social & 3 & 530 & & 8 & (i) \\
\hline TOTAL DEL. ESTADO & 8 & 2.919 & 235 & 14 & \\
\hline RAJA CALIFORNIA NORTE & & & & & \\
\hline \multicolumn{6}{|l|}{ Lisverstulad Autónoma ie baja California Norte } \\
\hline Esc. de Enfermeria (Mexicali) & 3 & 1.225 & & 3 & COD \\
\hline Fsc. de Medicina (Mexicali) & 3 & 630 & 120 & 6 & (DI) \\
\hline Fse. de Medicina Veterinaria y Zootecinan :Mexicali) & 4 & 6932 & & 59 & (1)1) \\
\hline Biblioteca Central (Tijuana) & 6 & 96 & 138 & 17 & CD! \\
\hline Fic. de Ciencias Quimicas (Tijuand & 4 & $5(x)$ & & 2 & ¿vb \\
\hline 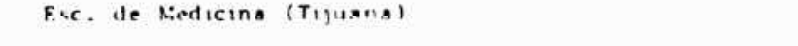 & 2 & 1.000 & 15 & 4 & NI.M \\
\hline TOTAL DEL ESTADO & 22 & 4.203 & 273 & 91 & \\
\hline \multicolumn{6}{|l|}{$\begin{array}{l}\text { RAJA CALIFOINIA SUR } \\
\text { \%o tiene }\end{array}$} \\
\hline TOTAL DE LA REGION & 39 & 13.614 & 7114 & 244 & \\
\hline
\end{tabular}

BiBL.TOTFCAS QIE SE ENCUENTRAN EN LAS INIVERSIDADFS REGIUN II

PERSONAT. LIERC3 VOI.S., ENLUAU. TE REV. CIASIF.

COAHIIILA

Universidad Autónoma de Coahuila

E.sc. de lice. on Enfermiria (Saltullo)

Esc. de Medicind linidad saltullo (Saltillo)

Fat. de Ortantologia (Saltillo)

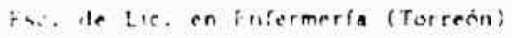

TOTAI. DEL. FETADO

\begin{tabular}{rrr}
1 & 1.345 & 714 \\
4 & 1.087 & \\
$\because$ & 787 & 1.345 \\
1 & & \\
\hline 8 & -1.219 & 2.059
\end{tabular}

Por Ma

TAMAULIPAS

Hniversilat Autonoma is Tamautipas

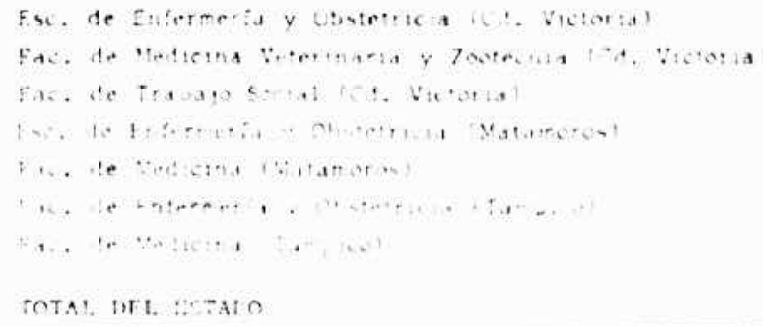


DIMLIOTECAS OUE SE EAEUENTRAK EN AS WEIWKAIDARES REGICN I

CHIIEAHUA

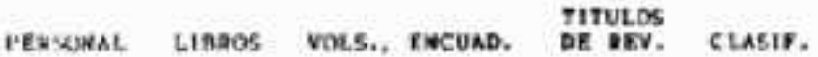

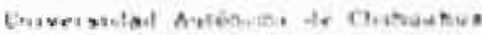

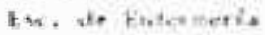

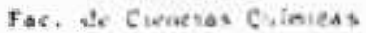

\begin{tabular}{ll}
7 & 1.300 \\
$x$ & 2.691 \\
2 & 2.135 \\
\hline 7 & 6.016
\end{tabular}

200

4

C1Bb

Fas. 40 simsticiats

TOTAL DEL ESTANO

ม.จกต A

Heaversulad de Sosora

twe de fonfecterin y feobajo Sestal

TOFAL DEL ESTADO

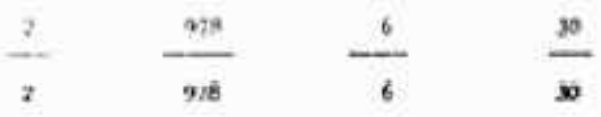

$\cos$

SINALAR

Imowersitial Autbonama de Stnatoa

Exe. de Ciensias det Mar

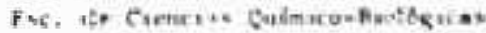

fors. Ir Trabaje sevial

TOTAL DEL. ESTADO

TAlA CATIROENIA NORTE

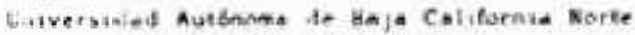

Ex, de Enferearia inextealy)

E.r. Je Mriticane (Mrasobl)

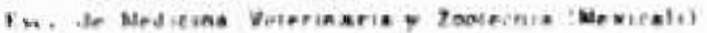

Biblioteca Centra\} ITiguana)

Tue. Me Cuencias Guinizat itiguena)

Eve, te Nedicana (Tijuxwer)

TOTAL DKL ESTADO

\begin{tabular}{rr}
7 & $\begin{array}{r}259 \\
1\end{array}$ \\
3 & 1,500 \\
5 & 530 \\
\cline { 2 - 2 } & 2,919
\end{tabular}

225

6

CBम

8

$3 \quad 1,275$

3630

2 tan

$6 \quad 96$

450

$1 \quad 1,000$

22

235

120

138

138

$\frac{15}{273}$

\begin{tabular}{|c|c|}
\hline 3 & $\mathrm{COD}$ \\
\hline 5 & Ctody \\
\hline 59 & t Elt \\
\hline 17 & $\operatorname{st} \theta$ \\
\hline$z$ & xub \\
\hline 6 & NL. \\
\hline
\end{tabular}

RApA CALITORuIA SUE

Tia thene

TOTAL, DE LA NGICK

39

13,614

714

246

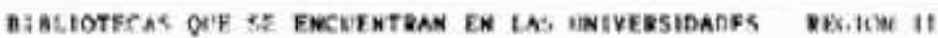

CoMHULA

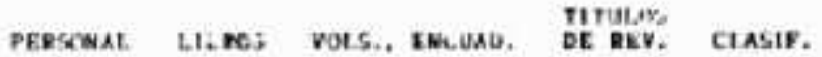

Enuversildad Authoma Ar Ceahulta

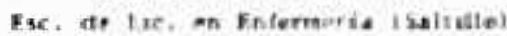

Fus, st wedicana tensind satritio (Satisile)

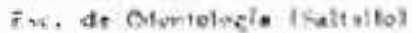

$1 \quad 1.365$

4. 1,087

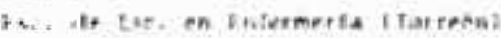

TOFAL DEL ESTASO

716
1,963

19

$\frac{1}{3}-\frac{1}{3,219} \quad \frac{}{2,059}$

TAMAULIFAS

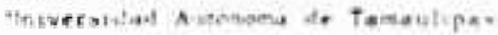

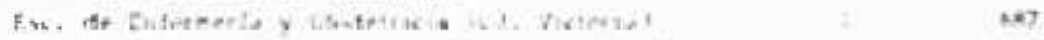

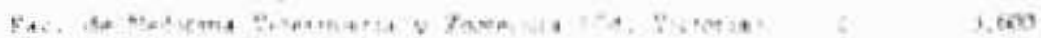

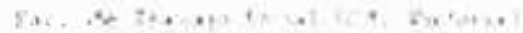

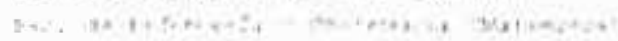

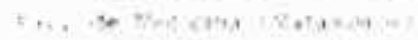

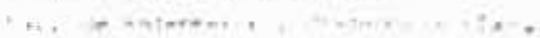

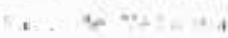

ror ins 


\section{GIMLIOTECAS QUE SE TMCURNTEAN EN LAS UNIVERSIDADES REGION IV}

\section{MAYARIT}

No tiene

COLIMA

No tuene

MICHOACAM

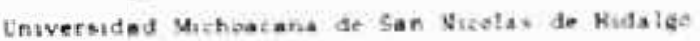

Esc a de Medacina

Esc, de Medicina Feterinaria $y, 7$ ovecnis

Fac. de ketestologia

Ared de sabud

TOTAL DEL ESTADC

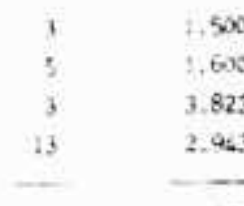

269.966

\section{GUANAJUATO}

tniversided de Cuanajuato

Ese, de Enfermeria y Obotetricia fétsva)

Esc. de Agronsmía y Zoctecnta (Itapuato)

Fac. de vedisina itebnl

\section{TOTAL DEL ESTADC}

\section{JALISCO}

Liniversidad de Guadalajara

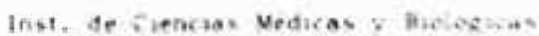

Fac. de Medicina

Fac. de cotontologla

TOTAL OEL ESTABO

\begin{tabular}{|c|c|c|c|}
\hline & $t .376$ & & \\
\hline & 100 & & 30 \\
\hline 6 & 5.000 & 3,050 & 130 \\
\hline & 5. 176 & 3,050 & 160 \\
\hline
\end{tabular}

TOTAL, DE LA EEGION

$22 \quad 3.30$

$10 \quad 0,5 x$

1. 3, , a

38

68
18,706

34,745

$\begin{array}{r}60 \\ 105 \\ 1 \\ \hline-\end{array}$

$$
83
$$$$
31
$$

122
CDD

LC

CDD

CDD

CDO

BIBLIOTECAS QUE SE EACURNTRAM EN LAS JMIVERSIDADES WEEIOW Y

PERSONAL Libros vols., EnCUAD. DE REV. Clasif.

\section{GUERRERO}

tiniversidad Autencen, de Gurerere

Esc. Superior de Medicina

TOTAL DET ESTADO

2. $\frac{\cos }{605} \frac{200}{200}$

PUEBLA

No trene

ESTADO DE MFXICO

Instutute Pobiecrice Nactonat

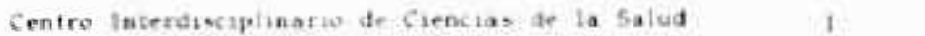

Universidad auténems del Futade de Mran.

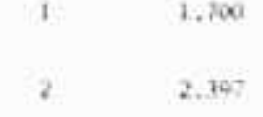

$20 \quad 13,000$

6.517
Ese. do Entermeria y Chastetric is

Universidad Naionat Auronona de Mexice

ENEP - Irtacata

FES: Cuatisian

TOTAL DEL ESTADO

s. (x)

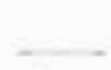

8,000

MORELOS

Uniwersidad Nacronal Aotenoma de Mavis

Centro de lav, robre fuacion del Nirrogene

TOTAL DEL. ESTADO
31

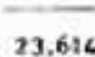

$\frac{7}{2} \quad \frac{625}{625}$ $\frac{46}{46}$
3

$\operatorname{con}$

$\cos$

tS

62

426 is

LE

te

1,200

1.482 


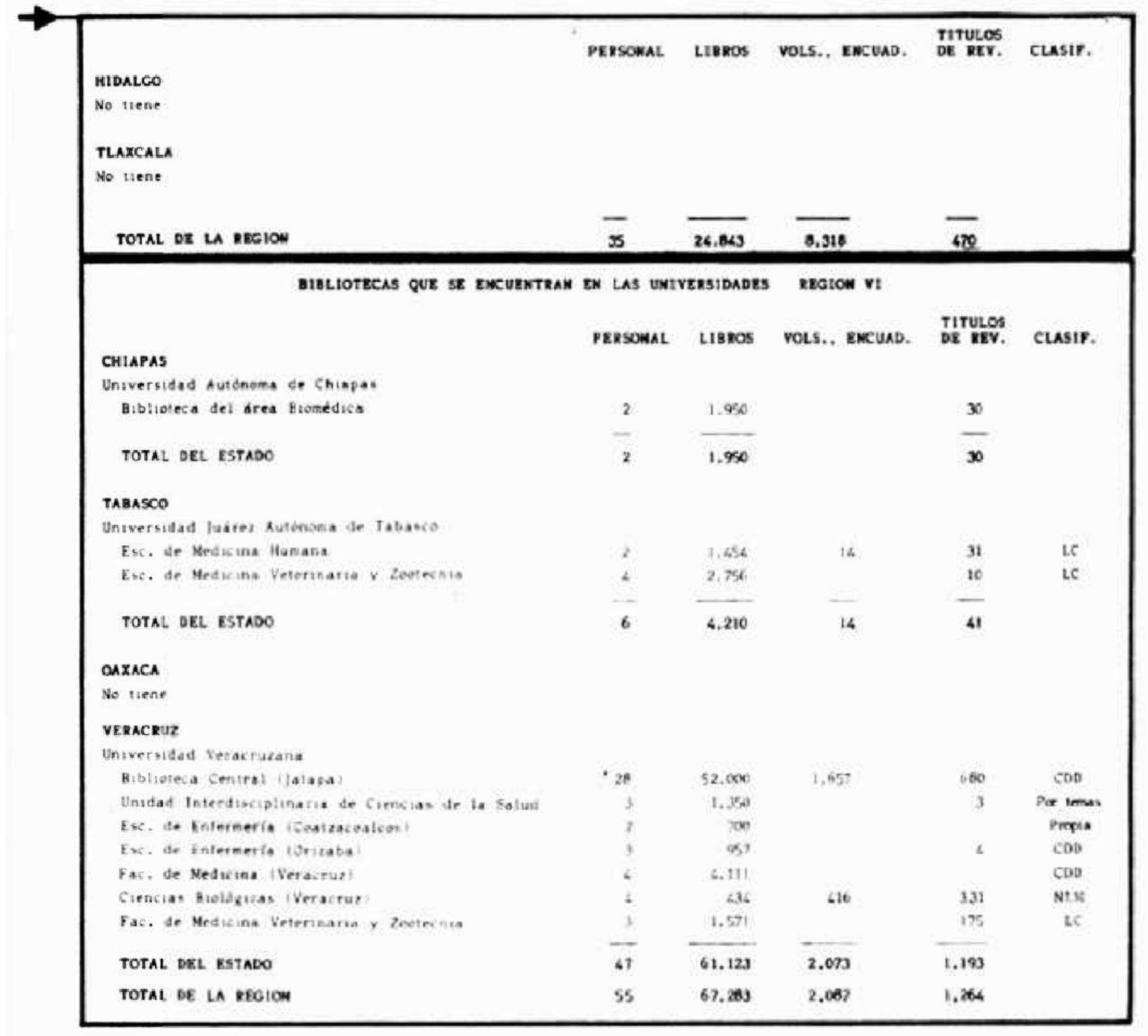

BIBLIOTECAS COE SE EHCUEMTEAN EN LAS UNIVERSIDADES REGIOW VII

PERSONAL LIBKOS vOLS. ENCUAD, DITULOS OEV. CLASIY.

CAMPECME

No tiene

QUINTAMA rox

No tiene

YUCAтAH

Unversidit be rusatin

Esc. de Nedicina Veterinaria o Zaetecara

Ese. de Enfermeria

\begin{tabular}{|c|c|c|c|}
\hline 3 & $x .900$ & 158 & 300 \\
\hline 1 & 318 & & 9 \\
\hline
\end{tabular}




\begin{tabular}{|c|c|c|c|c|c|}
\hline Es5, de Medicina & 4 & 3,560 & 66 & 20 & CDD \\
\hline Esc, de Quimica & 2 & 2,021 & 17 & 17 & $\operatorname{CDD}$ \\
\hline Biblioteca "Dr. Rogelio Dian Euerecto" & 1 & 2.162 & 405 & 16 & $\mathrm{CDD}$ \\
\hline Fac. de odontalogía & 2 & 839 & & 16 & CDD \\
\hline TOTAL OEL ESTADO & 13 & 12.240 & 660 & 386 & \\
\hline TOTAL DE LA REGIOM & 13 & 12,260 & 620 & 384 & \\
\hline
\end{tabular}

BTBL TOTECAS QJE SE ENCUENTKAN EN LAS UNIVERSIDADES REGION VIII

PERSCNAL IIBRO5 vOt.5., EMCUAD. TE DEV. CLASIF.

DISTRITO FEDERAL.

Instatute Polutenice Nacional

Centro de Investidacion $\gamma$ Entudies

Avanzados. hibliahrmeroteca

Departamento de Risquimua

Depastamento de Biolecnologis $\gamma$ Bioingeniefia

Departamento ise farmacologla $\gamma$ Texicología

Departamento de Investrgación Edocativa

Eso. de Enfermeria y abueteriata

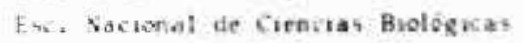

Ese. Vaciomal dir Vefreina flomeopetua

iese, Superrar te Medusina

the de Enfermería Escandion

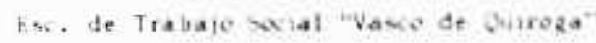

ISSSTE

Esc. de Enferneriat

IMSS

Fse- de Fnfermería*

Secretaría de la Defensa

Ess. Medico Mulirar

Secretaría de Salubridad y Asistencis"

Ese. de solud Fublica de Mexice

U.A.M

Xechimilco

Universidad Femenina de México

1

2

21

4

4

Universidad Nacional Autónema de Wexice

Centro de Investigación y Servicios Educatives

Direceión de Servicios Médicos

Esc. Nacapnal de Enfecmeta y Obstetracsa

Esc. Nacional de Trabajo Social

Fac, de Hedicina

Depto, de Histeria y rilosalta te la Mrdicina

Depto. de Psicologin Medica. Psiguiatria y Salud Mental

Div. de Iavestigación

Fac. de Mrdicina Veterinaria y Zootecnusa

Fac, de Odoniologis

Far. de Parcología

Fac. de Quimica Dis. At Estadias de Posgrado

Insibtuto de Biología

Departamento de Botinsea
$4,5,5,12$

1.597

$\therefore$. 095

10,124

3,152

tiones:

1.400

10,000

2.397

600

1.543

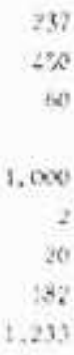

\begin{tabular}{|c|c|}
\hline 1.803 & 16 \\
\hline $16 \mathrm{C}$ & Propla \\
\hline 53 & 1.5 \\
\hline 96 & 16 \\
\hline$y S_{C}$ & $C D D$ \\
\hline 11 & $\therefore D_{1}$ \\
\hline 120 & (D) \\
\hline 10 & $\therefore 0$ \\
\hline \multirow[t]{2}{*}{20} & $\therefore \mathrm{D}$ \\
\hline & $C D D$ \\
\hline 6 & $\therefore \mathrm{DO}$ \\
\hline
\end{tabular}

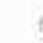

7f)

25

37

10

6.110

250

1.500

64

6.465

16.100

300

L. 567

7.873

7,500

17.000

1. 334

5.500

6. 000

c. 1.3

3.000

2.420

90

3,000
2,610

7

I

322

3, 001

(a)

1. 009

5. 000

is

345

7,0 tie

3. 500

10.000
LC

NI M

SDD

5

6

D.)

ma

vo

DD

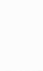

CDD

CDD

LC

DD . LS 


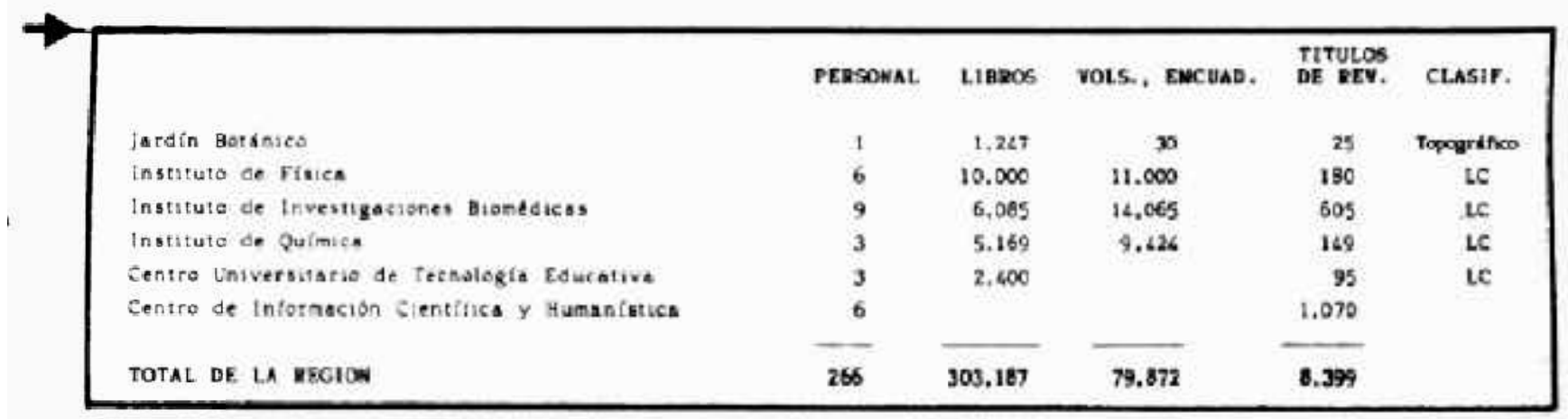

TOTAL DE BECURSOS DE LAS BIBLIOTECAS OFICIALES

\begin{tabular}{|c|c|c|c|c|c|}
\hline & $\begin{array}{l}\text { N* DE } \\
\text { BIBL IOT. }\end{array}$ & PFQSOMAL & LIBRO5 & YOLSF., EACUAD. & $\begin{array}{l}\text { TITULOS } \\
\text { DE NEV, }\end{array}$ \\
\hline $1 \times 55$ & it & $|5|$ & $52, \infty 1$ & $67,8 \times 6$ & 7,163 \\
\hline ISSSTE & ? & 3) & 16,314 & {$[0,393$} & 1.168 \\
\hline $55 A$ & 12 & jo & 41,917 & 33.419 & 2,236 \\
\hline Total. & T & 258 & 108.592 & 91.396 & 10.545 \\
\hline
\end{tabular}

IMSTITUTO MEXICANO DEL SEGUNO SOCIAL

\section{AGUASCALIENTES}

Clinsa Hesprtal $T^{-1}$ sum.

\section{BajA CALIfORNIA NORTE}

Clinas hosplial wrat. de Zena 11 yom.

clinuca Hespitel 1 i sivim. if

Hespita! Eenera!, Zna 111

CHIHUAHUA

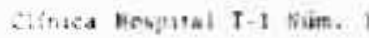

COAHUILA

clinted Hespital $T$, n kim.?

elínuca Hospula) t-2 kán. ?

Clinse Hexp:ut T-i Kidt. 16

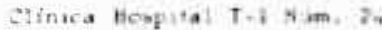

DISTRITO FEDERAL.

Bebloteca Central

B.tisteva de mgurtada rox.at

Fsc. de Futermeria L.M.N.-

Hosprtal de surdiologin y Neumolozis

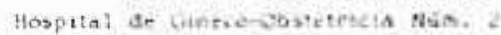

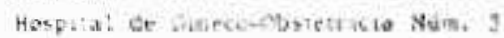

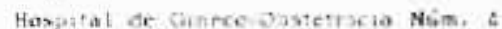

Hespital de Murologia

\section{PERSCWAL LIBNOS}

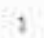

1

2

3136

5.39

2

663

2200

$1 \quad 156$

230

1 RF

236 vols., Encuad.

TITULOS

DE REV. CLASIE.

556

267

Nis

65

617

$10 \%$

51.

MLM

s1. $M$

150

961

86

463

$\begin{array}{cc}60 & \text { sLM } \\ 56 & \text { sLM } \\ 56 & \text { sL\% } \\ 100 & \text { Toxwst? }\end{array}$




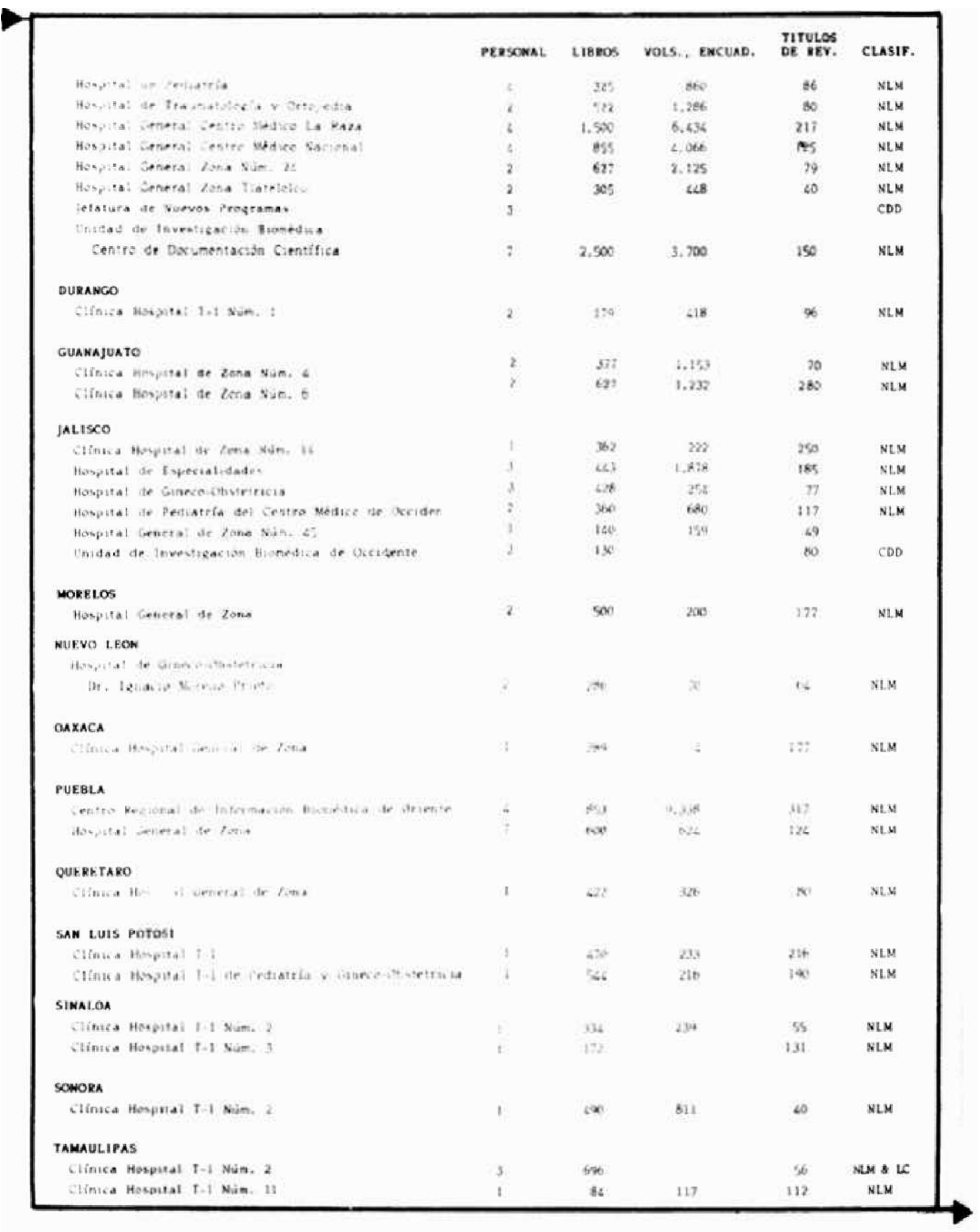




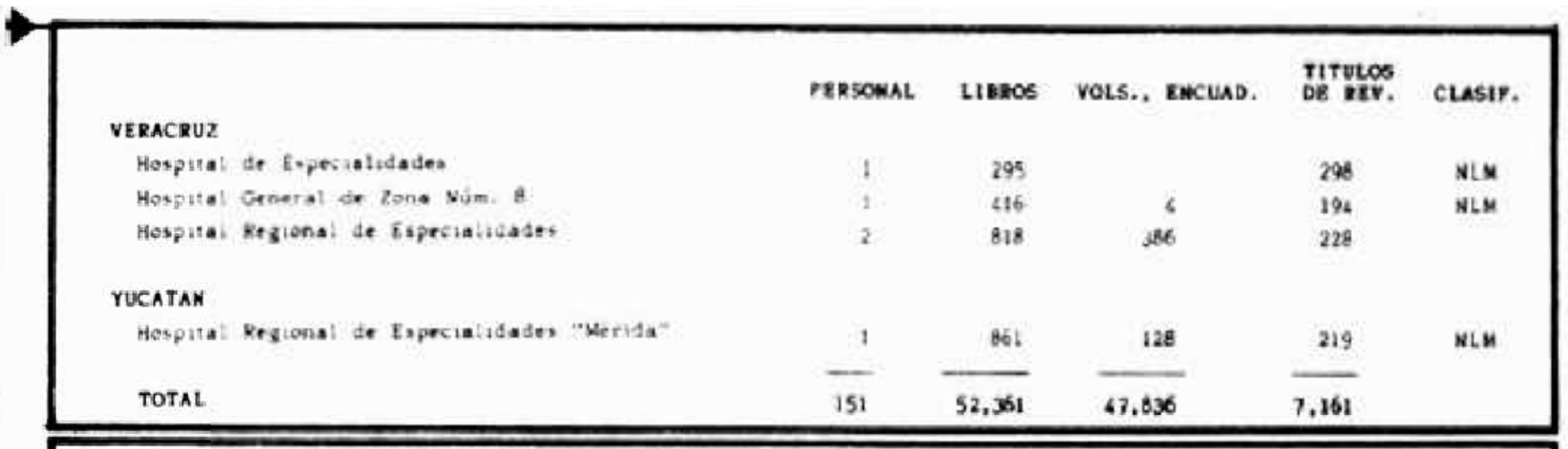

INSTITUTO UE SEGURIDAD Y SEFVICIOS SOCIALES DE LOS TRABAIAWORES DE. ESTADU
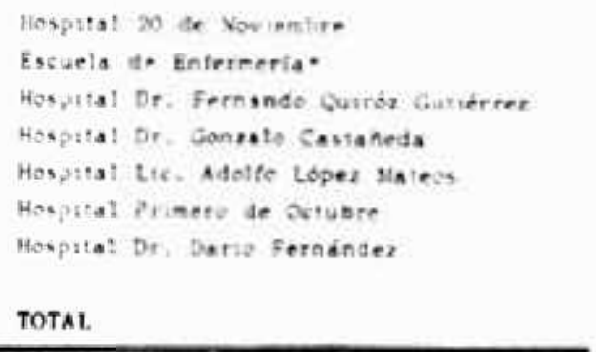

SECRKTARIA DE SALUERIEAO Y ASISTENCIA

\begin{tabular}{|c|c|c|c|c|}
\hline in & 7,262 & 6,323 & 500 & LC \\
\hline I & 1,593 & & 5 & Le \\
\hline 3 & 1.660 & 340 & 35 & LC \\
\hline 2 & 758 & 495 & 145 & te \\
\hline 2 & 1, C43 & 2.879 & 191 & te \\
\hline 6 & 1.250 & 150 & 178 & NLM \\
\hline 2 & 1.000 & 152 & 96 & te \\
\hline- & $-\cdots$ & - & - & \\
\hline 37 & 16.316 & 10,339 & 2.148 & \\
\hline
\end{tabular}

\begin{tabular}{|c|c|c|c|c|c|}
\hline SECRKTARIA DE SA & UERItano $Y$ & ASISTENCIA & vats., RACUAD & $\begin{array}{l}\text { TiTuLos } \\
\text { DE REV. }\end{array}$ & CLASIF. \\
\hline \multicolumn{6}{|l|}{ Derescion Cimeral de Setvicies Sooctinados } \\
\hline De Salud cublica en low Erados & 2 & 2.125 & 250 & 177 & Evis \\
\hline Hesplad have? & 2 & 3.500 & & 250 & $\begin{array}{l}\text { Win. Progre } \\
\text { 4wo }\end{array}$ \\
\hline Escuels te Soluif Publicen te Mexscot & 11 & 10.2000 & c.110 & 250 & $\cot$ \\
\hline Hospatal General & 8 & 6.506 & & & $\operatorname{CDO}$ \\
\hline $\begin{array}{l}\text { Instututo de Selubridad y Entermedades Tropicatev } \\
\text { Instututo Wasienal de Cardialozis }\end{array}$ & $y$ & 3.698 & & 660 & $\cos$ \\
\hline Dr, Ignecto Chiver & 5 & 9.169 & 13,993 & Sot & * $\mathrm{M} s \mathrm{LC}$ \\
\hline Instituto Hecioeal de Xeuralogla & 3 & 2.000 & 10,000 & 200 & $\cos \theta$ \\
\hline Subsecrebria de Negoramients det anbiente & 4 & 3.00 & 5.000 & 50 & cos \\
\hline hes,pral Geners) A* (Nershuatceyotil & $z$ & 118 & 13 & 28 & CDs \\
\hline Instutute Nacienal de Canserolozía & $z$ & 1,293 & $5 s$ & & Kinguns \\
\hline Hospital Cenerol de Oxeidente & 2 & 120 & & 100 & LC \\
\hline 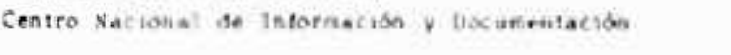 & 23 & & & & \\
\hline TOTAL & 20 & 61.917 & 33,619 & 2.236 & \\
\hline \multicolumn{6}{|c|}{11} \\
\hline 4 & PEBSONAL & Eireos & VOLS., EACUAD. & TITULOS & CLASIF: \\
\hline \multicolumn{6}{|l|}{ DISTRITO FEDREAL } \\
\hline Asociación Dental Mexicana. A.r. & 4 & $3,0<2$ & 290 & 35 & CDD \\
\hline Aroxiacion Mtdice Hoppatat X.B.C. & 2 & 250 & 90.3 & so & NLM \\
\hline Asociación Mtxicent de Aceubn cenira is Lepra, A.C & 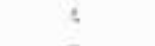 & 1.427 & 6.30 & 196 & $\cos$ \\
\hline Asociacion Pro-satud uaternal. A.C. & 2 & 6,000 & 3.000 & 10 & CoD \\
\hline Hespatal General "Dr. Wanuel Gea Gonzater" & 5 & 869 & 2.110 & 78 & CDD \\
\hline
\end{tabular}




\begin{tabular}{|c|c|c|c|c|c|}
\hline tesplta: Infantetl de Vêxice & 2 & 9.538 & 10.561 & 475. & $C D D$ \\
\hline ANFOTES & 17 & 4.000 & & 500 & $C D D$ \\
\hline Invitide Mexicans de Psiquiatris & 5 & 2.064 & & 140 & Propia \\
\hline Instrite Nactonal de Enfermedades Pulmonater & $\therefore$ & 100 & 700 & 26 & Propla \\
\hline Instatute Nacional de Nutrición & 6 & 2,700 & 14.000 & 209 & CDD \\
\hline \multicolumn{6}{|l|}{ Laboratoria Nacionales de forento industial } \\
\hline ICentro Nexicano de Información CWimla & 16 & 2,800 & 860 & 165 & $\mathrm{CDD}$ \\
\hline Organización Mundial de ta Galue & 2 & 5.000 & 250 & 5 & Propia \\
\hline Bublioteca de Ganader'a ISARHI & 2 & 8,000 & 25 & 25 & \\
\hline Durecciôn de Sanidad Animal (SARH) & 1 & 900 & 600 & 3 & Alfabeitiea \\
\hline Instututo Nacional de Investigaciones Pecuarias & 15 & 7.500 & $3 . \angle 00$ & 505 & LC \\
\hline Hosplta! de la Sectetaria de Hacienda y Credito Püblieo & 3 & $52 !$ & 127 & 44 & NLM \\
\hline Hospital Central de la Secretaris de to Defensa Mihtar & & 3.000 & 2.352 & 56 & CDD \\
\hline Secretaria del Tratiaja y previsión Secial & \& & 20.000 & & 300 & CDO SRO1 \\
\hline \multicolumn{6}{|l|}{ Sistema Naciona! para el Desarrollo Integral de la } \\
\hline $\begin{array}{l}\text { Familia (DIf) } \\
\text { Insturato Nactonal de Perinatologia (D) F }\end{array}$ & 5 & 430 & & 122 & Num. Progre \\
\hline \multicolumn{6}{|l|}{ Socredad Benetled Española } \\
\hline Hoxprta: Fspanol & 2 & 250 & 500 & 150 & Propia \\
\hline Sociedad Medica del Hospital General, 5.5, A. & ; & & 7.500 & 125 & $C D D$ \\
\hline SYNTEX. C, A. & 1 & 1.200 & 2,500 & 40 & $C D D$ \\
\hline Centros de thegración fuvetint. A. C. & $: 7$ & 3.000 & & 200 & Adap. ARF \\
\hline TOTAL DE LA REGION & 148 & 85.591 & 56.650 & 3.761 & \\
\hline TOTAL DE TODAS LAS REGIONES & 170 & 100.871 & 58,555 & 4,788 & \\
\hline
\end{tabular}

INSTITUCIONES DIVERSAS POR REGION

PEFSONAL L.IBROS vOLS., ENCUAD. TE REV. CLASIF.

REGION I

BAJA CALIFORNIA SUR

ientro de Investigaciones buslogicas de Baja Galforna, A. C.

600

40

CDD

JALISCO

Hospital Civi! le ifuadalajara

4280

REGION IV

3,000 $142 \quad$ TEMAS

REGION V1

CHIAPAS

Centro de Investigactones del Sureste

$8 \quad 7,000$

200

$\mathrm{CDD}$

VERACRUZ

Instituto Nacional de Investigaciones sobre Recursos Bióticos

TOTAL. DE LA REGION

5
13 $\quad \frac{5.500}{12.500}$

205

LC

205.420

REGION VII

YUCATAM

Centro Nacional de investigaciones Agricolas

de la penirisula de Yucatan

$3 \quad 1.900$

900

423

CDD 